Proceedings of the Operational Research Society Simulation Workshop 2021 (SW21)

M. Fakhimi, D. Robertson, and T. Boness, Eds.

DOI: https://doi.org/10.36819/SW21.008

\title{
AGENT-BASED MODELS: A TUTORIAL
}

\author{
Dr Duncan A. Robertson \\ School of Business and Economics \\ Loughborough University, Loughborough, LE11 3TU \\ d.a.robertson@lboro.ac.uk \\ http://www.duncanrobertson.com
}

\begin{abstract}
We introduce agent-based modelling in this tutorial paper. We introduce the concepts of agent, emergent behaviour, and show these concepts in three different agent-based models.
\end{abstract}

Keywords: Tutorial paper, agent-based modelling, techniques

\section{INTRODUCTION}

Agent-based modelling ('ABM'), or multi-agent simulation, is a technique where individual 'agents' are modelled, where the behaviour of these agents combine to make up the overall system. This micro-level modelling can be contrasted with modelling stocks and flows in system dynamics ('SD') models or modelling systems as a series of events in discrete event simulation ('DES').

While agent-based modelling is somewhat intuitive, in that interactions between individual agents is the fundamental building block of the modelling technique, agent-based modelling still requires a degree of high-level computer coding rather than using drag-and-drop building blocks to create a model.

\section{AGENT-BASED MODELS: FUNDAMENTALS}

Agent-based models are a form of simulation, where individual components of the system are modelled. However, these components - or agents - can have autonomy. They can take into account the environment, or the interactions with other agents, in order to allow their behavior to adapt. Unlike SD models, where the movement of objects through a system is not explicitly modelled, ABM uses these agents as the building blocks of the simulation: it is a bottom-up rather than top-down methodology. And unlike DES, agents within an agent-based model can have autonomy rather than being directed through a system by a controller.

This tutorial will introduce three agent-based models to show the fundamental building blocks of an agent-based model.

\subsection{The Agent: Autonomy}

Agents are the fundamental components of agent-based models. These are (or can be) autonomous, in that their behaviour is controlled by the agent themselves, rather than being directed: they can 'think' for themselves. As such, the use of agent-based models has been used by researchers in several disciplines to model social behaviour as well as the behaviour of physical systems. 


\section{Robertson}

'Thinking' in an agent-based modelling context is a term that encompasses the ability of agents to respond to stimuli; it does not necessarily mean that the agent has cognitive ability. Take for example the interaction of three masses that experience gravitational or electrostatic attraction to each other. In each case, the bodies - or agents - follow forces proportional to the inverse of the square of the distance between the objects. In an agent-based model, the direction of the force vector could be computed by each agent and the resulting direction of movement calculated. In this case, however, the agents do not have cognition, they merely respond to the force vector to which they are exposed: the masses do not know the location of the other masses, and therefore cannot be expected to take this into account when reacting to their position. Agent-based modelling is concerned with modelling the social, physical, psychological, behavioural 'forces' that affect the state or actions of an individual.

Agents within agent-based models are autonomous, in that their behaviour is modelled as being controlled by the agent themselves without any over-arching controller of the system: in this way, the macro-level model behaviour in built up from the individual actions of the agents that collectively comprise the system.

\subsection{Emergence}

An early use of agent-based models was to model the spatial interaction of birds. One can observe the phenomenon of flocking in bird populations, but the modelling of this using conventional simulation techniques is difficult. Reynolds (1987) attempted to model the flocking behaviour not by modelling the entire flock, but by simulating each individual bird. These birds do not have access to information about the position, speed, and direction of each bird in the population, but only about their local neighbourhood, parameterized by a radius and angle of observation. By imposing simple rules on the behaviour of individual birds, namely separation (steer to avoid other birds); alignment (steer towards to the average heading of birds within the local neighbourhood); and cohesion (move towards the position of birds within the local neighbourhood), 'lifelike' flocking behaviour is simulated.

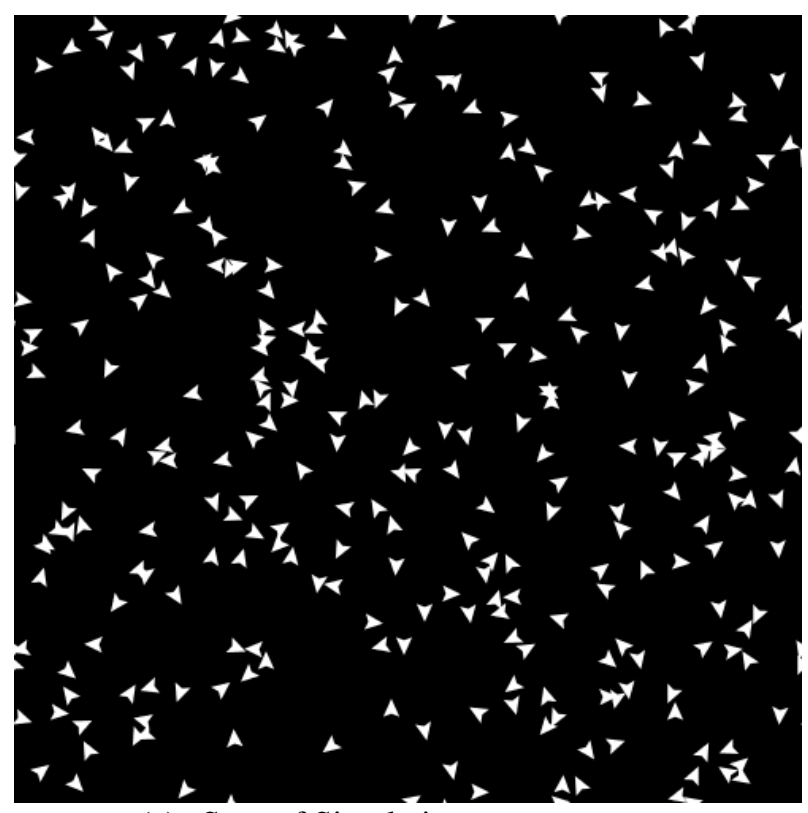

(a) Start of Simulation

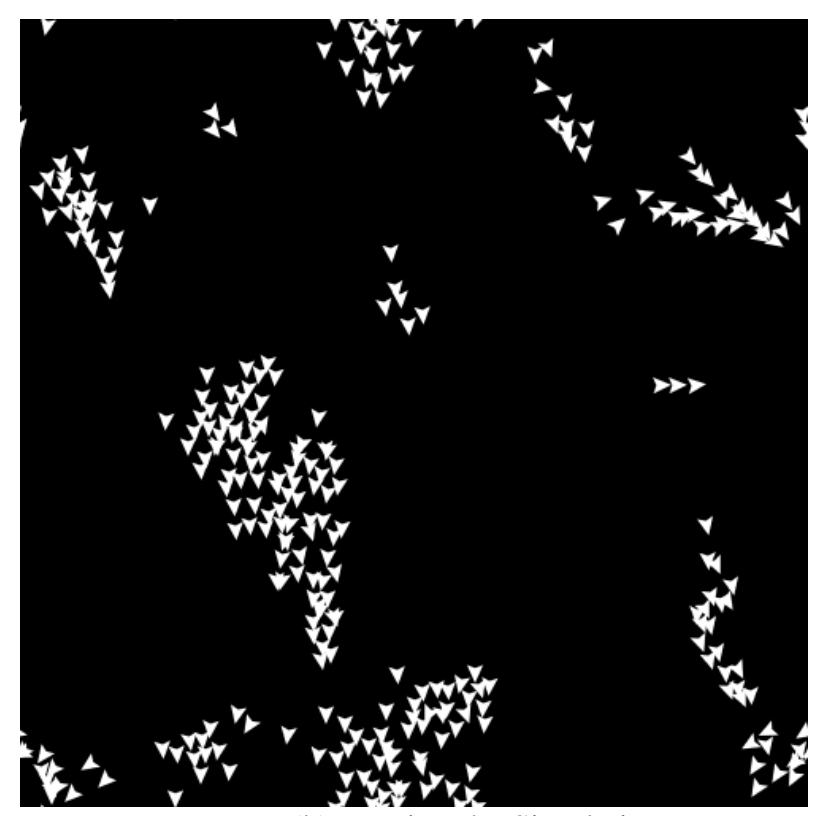

(b) During the Simulation

Figure 1 Flocking Model showing Emergent Flocking Behaviour

In Figure 1, birds are initially positioned at random on the space (note that the space wraps around so that agents can spill over from the left to the right; from the top to the bottom, or vice versa - this is a torus, a 


\section{Robertson}

common topology for agent-based models in that it avoids hard boundaries and the necessity of modelling what happens at these boundaries). These bird agents then follow simple rules that take into account their local observations, which then leads to the system-wide emergent property of flocking.

\subsection{Bounded Information}

In the flocking model above, agents need not (and generally do not) have full information about the state of the system: they tend to be myopic, in that their knowledge of the system is local. This leads to boundedly rational behaviour (i.e. their behaviour may have been different if they had full information). As such, this can lead to interesting outcomes.

In Schelling's (1971) model of segregation - generally accepted as one of the first agent-based models, individuals in a city determine whether they are happy or unhappy based on the number of neighbours who are the same colour as themselves. Agents - who have a property of colour - are first arranged randomly on a grid. A model parameter determines the threshold at which agents are happy: each agent calculates the proportion of their neighbours who are a different colour to them: if this is above the threshold value, the agent moves to a vacant space, as shown in Figure 2
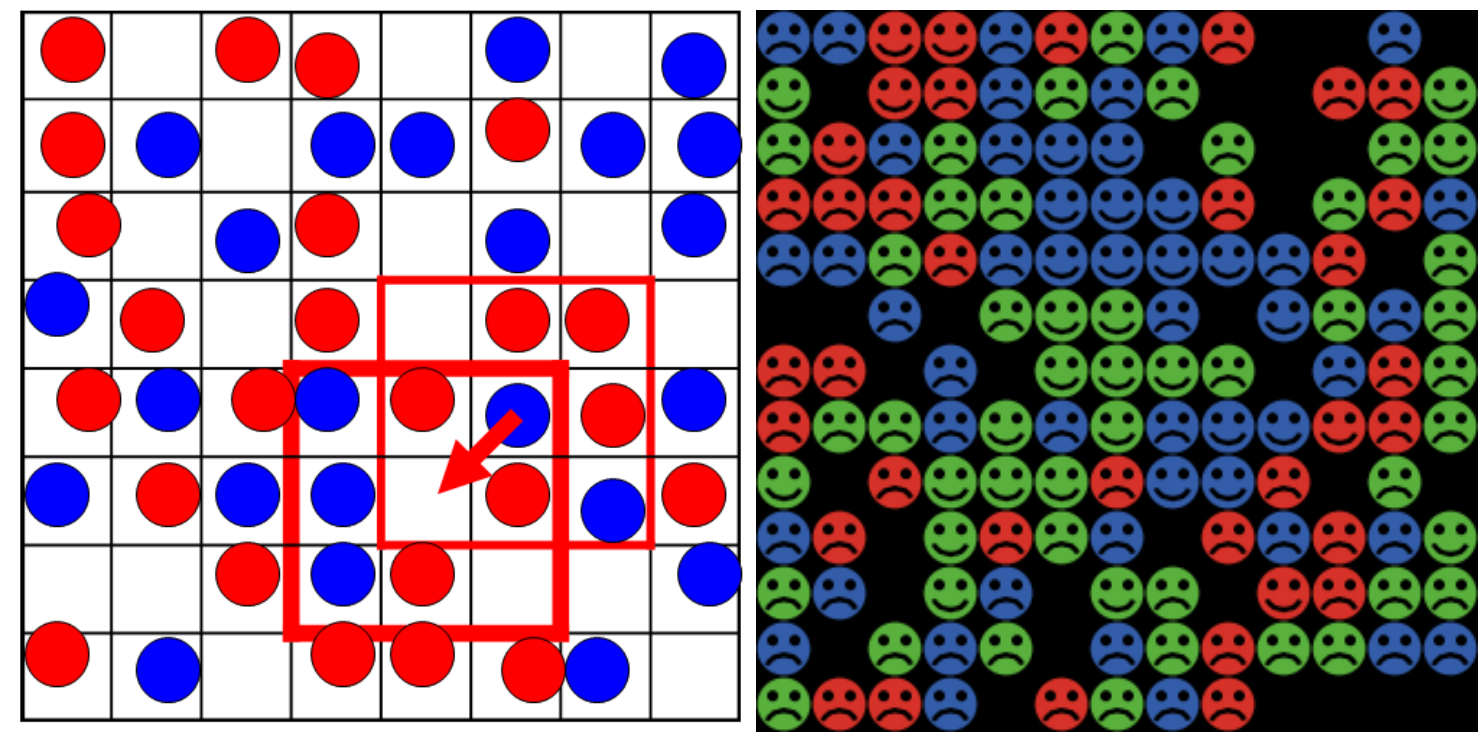

Figure 2 Schelling's Segregation Model (images from duncanrobertson.com)

Schelling's model is important in that it demonstrates that macro level behaviour can come from micro level interactions. In particular, the agents self organize into colour segregated clusters without the need of a central planner. In particular, if the threshold value is below $50 \%$, i.e. agents are happy to be in a local minority, segregation still forms. This behaviour cannot readily be modelled using traditional techniques.

\subsection{Applications in Management}

There are several applications of agent-based modelling in business. Within the strategy field, individual firms' strategies can be modelled, and the effect on the business landscape and other firms' strategies can be modelled. Robertson (forthcoming) and Robertson and Caldart (2010) set out a model of interacting firms by using an agent-based model to show how strategic movement of one firm deforms the fitness landscape which in turn alters the strategic movement of other firms. Robertson (2019) provides an overview of Agent-Based Models in Strategic Management 


\section{Robertson}
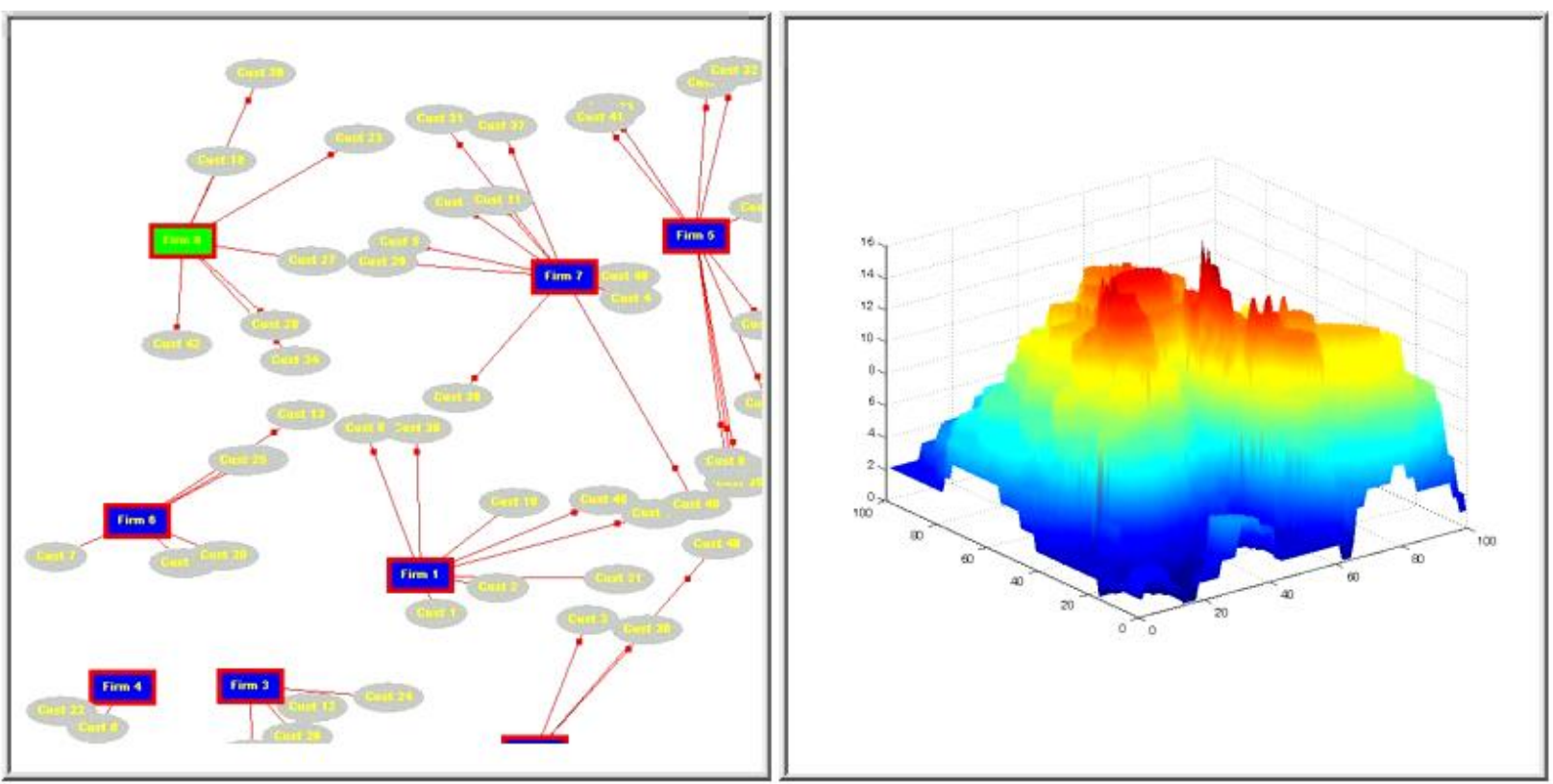

Figure 3 Robertson's Dynamic Competition Model (image from Robertson (forthcoming))

\section{CONCLUSION}

The concepts of emergence and self-organization are best demonstrated by the use of agent-based models. The models introduced in this tutorial other models will be introduced in the SW20 workshop, which is intended to be an interactive session where agent-based models can be developed interactively with workshop participants.

\section{REFERENCES}

Gilbert, N. and Troitzsch, K. (2005) Simulation for the Social Scientist, Buckingham: Open University Press

Reynolds, C. (1987) 'Flocks, Herds and Schools: A Distributed Behavioral Model', SIGGRAPH '87: Proceedings of the $14^{\text {th }}$ Annual Conference on Computer Graphics and Interactive Techniques. Association for Computing Machinery, 25-34

Robertson, D. A. and Caldart, A. A. (2010) The Dynamics of Strategy, Oxford: Oxford University Press

Robertson, D. A. (2019) Agent-Based Strategizing, Cambridge: Cambridge University Press

Schelling, T. C. (1971) 'Dynamic Models of Segregation', Journal of Mathematical Sociology, 1, 143186

\section{AUTHOR BIOGRAPHY}

DUNCAN ROBERTSON is a member of the Management Science and Operations group at Loughborough University and a Fellow of St Catherine's College in the University of Oxford. He received his undergraduate degree in physics at Imperial College London and his DPhil at the Said Business School, University of Oxford. Further information and details about agent-based modelling can be found at http://www.duncanrobertson.com/ 